\title{
LIBERACIÓN DE Chrysoperla argentina (NEUROPTERA: CHRYSOPIDAE) PARA EL CONTROL DE Trialeurodes vaporariorum (WESTWOOD) (HEMIPTERA, ALEYRODIDAE) EN INVERNÁCULO DE PIMIENTO EN TUCUMÁN, ARGENTINA
}

\author{
RELEASE OF Chrysoperla argentina (NEUROPTERA: CHRYSOPIDAE) FOR THE CONTROL \\ OF Trialeurodes vaporariorum (WESTWOOD) (HEMIPTERA, ALEYRODIDAE) IN \\ GREENHOUSE PEPPER IN TUCUMÁN, ARGENTINA
}

Gabriela Cecilia Flores, Carmen Reguilón, Germán Luis Alderete y Daniel Santiago Kirschbaum

\begin{abstract}
RESUMEN
Se hizo un estudio con el objetivo de regular los niveles poblacionales de la mosca blanca Trialeurodes vaporariorum (Hemiptera: Aleyrodidae), en un cultivo de pimiento (Capsicum annuum L.), bajo un invernadero en la localidad de San Isidro de Lules, provincia de Tucumán (Argentina), mediante el uso de Chrysoperla argentina (Neuroptera: Chrysopidae), el cual es un depredador polífago nativo. En el invernadero se establecieron dos sectores: módulo testigo (MTe) y módulo tratamiento (MTr); en cada uno de ellos se seleccionaron cuatro parcelas al azar de 20 plantas cada una y se realizaron monitoreos periódicos de mosca blanca en diez plantas enumeradas por cada parcela. Una vez que la densidad poblacional de mosca blanca alcanzó el umbral de daño económico, estimado en $>50$ adultos por planta, se efectuaron dos liberaciones de $C$. argentina en estado adulto y larval, en el MTr. Se evaluó el efecto de control de la crisopa sobre la mosca blanca. Los resultados fueron analizados estadísticamente mediante análisis de la varianza (prueba F) y prueba t. Se confirmaron diferencias significativas en cuanto al número de individuos de moscas blancas entre MTe y MTr, correspondientes al último muestreo, posterior a la liberación del depredador. A partir de este estudio se concluyó que $C$. argentina logró disminuir los niveles poblacionales de T. vaporariorum, por lo que es considerado un controlador eficaz de este fitófago.
\end{abstract}

PALABRAS CLAVES: control biológico, mosca blanca, crisopas, Capsicum annuum L.

\begin{abstract}
A study was conducted with the aim of regulating population levels of Trialeurodes vaporariorum (Hemiptera: Aleyrodidae) using Chrysoperla argentina (Neuroptera: Chrysopidae), a native predatory insect, in a pepper (Capsicum annuum L.) crop grown under a greenhouse in San Isidro de Lules, Lules Department, Tucumán (Argentina). The greenhouse was divided in two sectors: control module (MTe) and treatment module (MTr). In each one, four plots of 20 plants were selected. Whitefly weekly monitoring were performed in ten plants numbered for each plot. Once the whitefly population density reached the threshold of economic damage, estimated over 50 adults per plant, two C. argentina releases in adult and larval stages were conducted in the MTr. The effect of control of the green lacewing on whitefly was observed. The results were statistically analyzed by analysis of variance and t-test. Significant differences of whithefly populations were found between MTe and MTr after the last release of the predator. The conclusion of this study is that $C$. argentina reduces population levels of T. vaporariorum, resulting an effective biocontrol agent against this greenhouse whitefly.
\end{abstract}

KEY WORDS: biological control, whitefly, green lacewings, Capsicum annuum L. INTA Famaillá. Ruta Prov. 301, Km 32. (4132) Famaillá, Tucumán. Argentina. E-mail: alderete.german@inta.gob.ar; kirschbaum.daniel@inta.gob. ar (G.L.A) (D.S.K) 


\section{INTRODUCCIÓN}

Argentina es el principal productor sudamericano de pimiento (Capsicum annuum L.) con aproximadamente 13000 ha cultivadas y una producción de $120000 \mathrm{t}$ anuales (Fernández Lozano, 2012). En la provincia de Tucumán, este cultivo se sitúa en el corredor pedemontano occidental, caracterizado por inviernos suaves, donde el departamento Lules se presenta como la zona más importante de producción, con unas 40 ha de invernaderos destinados en su mayoría a la producción de pimiento. También se cultiva en los departamentos de Trancas, Famaillá y Leales pero en menor proporción (GL Alderete, com. pers.).

Las "moscas blancas" Bemisia tabaci (Gennadius) $y$ Trialeurodes vaporariorum (Westwood) son las principales plagas que afectan la producción de pimiento bajo cubierta plástica. Para la provincia de Tucumán y a nivel general, la especie Trialeurodes vaporariorium, es un insecto perjudicial de gran importancia, de distribución mundial y asociada a 249 especies de plantas (MAG Costa Rica, 2015). Afecta el crecimiento, transmite virus que provocan enfermedades, favorece el desarrollo de fumagina en hojas y frutos (Duffus, 1965; Ortiz-Catón et al., 2010; Sistema Nacional Argentino de Vigilancia y Monitoreo de Plagas, 2015) y, tal como se observó en la especie relacionada $B$. tabaci, es muy probable que la resistencia de T. vaporariorum a los plaguicidas se incremente con el transcurso del tiempo (Dennehy et al., 2005).

El control biológico es una estrategia utilizada mundialmente para el manejo de plagas, en cultivos tanto en campo como bajo invernaderos, que surgió como complemento del control químico, el cual tiene consecuencias tales como aparición de resistencia en las plagas, altos costos de producción, bioacumulación a través de la cadena trófica, polución del ambiente, pérdida de biodiversidad y riesgos para la salud (Bale et al., 2008). En este sentido, se observa como tendencia que los mercados exigen de manera creciente alimentos con menos residuos tóxicos y con menor impacto ambiental en la producción, lo que genera nuevas oportunidades para el empleo enemigos naturales para el control de plagas (Brimmer y Boland, 2003). En el grupo de enemigos naturales se encuentran parasitoides del Orden Hymenoptera, depredadores de los Órdenes Neuroptera y Coleoptera, y hongos entomopatógenos del género Cladosporium Link, los cuales actúan en la regulación de las poblaciones de plagas y consecuentemente disminuyen el daño en los cultivos (Ghiggia et al., 2003; Tapia et al., 2005; López et al., 2005; Fernández et al., 2009).

Los géneros y especies de la familia Chrysopidae, perteneciente al Orden Neuroptera, son depredadores considerados controladores biológicos decisivos para la regulación de insectos plagas (Breene et al., 1992; Gerling et al., 1997; Aybar-Guchea et al., 2010; Haramboure et al., 2014), y se han podido criar para su comercialización a nivel mundial (Tauber et al., 2000).

Existen antecedentes del uso de las Chrysopidae en el manejo de mosca blanca, con diversos resultados. En viveros de plantas ornamentales de Hibiscus rosa-sinensis, se evaluó el control de B. tabaci con Chrysoperla rufilabris. Se utilizó la presencia de fumagina como indicador, dando como resultando plantas comercializables al final del experimento a las tratadas con el depredador (Breene et al., 1992). Se realizaron estudios de la fluctuación poblacional de B. tabaci y de Chrysoperla carnea sobre Gossypium hirsutum L. (Gerling et al., 1997), los resultados mostraron que aunque las crisopas se reprodujeron en el campo junto con las moscas blancas y sus larvas fueron alimentadas con ninfas de éstas, $C$. carnea no fue un eficiente agente de control, probablemente debido al cumplimiento marginal de las demandas nutricionales y ambientales del depredador.

En pimiento y tomate (Solanum lycopersicum), se probó el efecto sobre B. tabaci de $C$. carnea, además de dos hongos entomopatógenos (Paecilomyces farinosus y $P$. javanicus), una barrera viva (Zea mays) y el insecticida sintético imidacloprid, se pudo observar que la combinación crisopa + imidaclorid, fue el tratamiento más efectivo en tomate (Ruiz y Medina, 2001). Sobre la mosca blanca Aleurotrachelus sociales Bondar y Sitotroga cerealella Olivier, se evaluó la capacidad depredadora de Ceraerochrysa claveri Navás (Velázquez-Grisales, 2004), se observó un incremento en el ciclo de vida utilizando como presa la especie A. sociales.

La especie Chrysoperla argentina ha sido encontrada en cultivos de pimiento bajo invernáculo en Argentina (Haramboure et al., 2014) y el conocimiento de su biología es avanzado (Reguilón et al., 2006). Estudios realizados en Tucumán, dan cuenta de la capacidad de depredación de $C$. argentina sobre diferentes plagas de los cultivos hortícolas de la región, entre las que se mencionan los trips y la mosca blanca B. tabaci (Ávila 
et al., 2009; Macián et al., 2009; Reguilón et al., 2014). Si bien hasta el momento no se evaluó la capacidad de control de la mencionada crisopa sobre T. vaporariorum, se presume que podría consumir todos los estadios biológicos de esta plaga, tal como se ha observado que sucede con otra especie de mosca blanca, B. tabaci (Ávila et al., 2009).

El control biológico de moscas blancas en pimiento es considerado como una de las alternativas más promisorias en Tucumán, en correspondencia a dos situaciones claves que se observan en el resto del mundo en los últimos años, tales como el incremento de la presencia de la plaga y las crecientes restricciones sobre el control químico (Bisset, 2002). En consecuencia, sería importante contar con información local sobre la dinámica poblacional de este fitófago y evaluar la posibilidad de influir sobre el mismo a través de la acción de enemigos naturales. En este contexto, el objetivo de este trabajo fue evaluar el efecto de la liberación de $C$. argentina como controlador biológico de T. vaporariorum en cultivo de pimiento bajo cubierta.

\section{MATERIALES Y MÉTODOS}

El ensayo se realizó entre los meses de septiembre y diciembre de 2010, en un invernadero tipo parral de Almería (Figura 1), de $20 \mathrm{~m}$ de ancho por $30 \mathrm{~m}$ de largo $\left(600 \mathrm{~m}^{2}\right)$, cultivado con pimiento, en etapa de cosecha. El invernadero está ubicado en la localidad

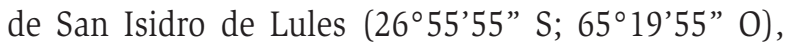
provincia de Tucumán (Argentina). Las variedades de pimiento evaluadas fueron "APL-82", "APL-11" y "APL-04", plantadas en abril del mismo año. En este invernadero se plantaron 1080 plantas (con un marco de plantación de $1,20 \mathrm{~m}$ entre camellones y $0,40 \mathrm{~m}$ entre plantas), repartidas en dos sectores o módulos de $10 \mathrm{~m}$ de ancho y $30 \mathrm{~m}$ de largo, designados como testigo (MTe) y tratamiento (MTr). En este último se realizaron las liberaciones controladas del agente de control biológico. Se registraron las condiciones de temperatura y humedad dentro del invernáculo.

Las 540 plantas de cada módulo se distribuyeron en 27 filas de 20 plantas cada una. Se delimitaron cuatro parcelas experimentales de una fila (20 plantas cada una), aleatoriamente distribuidas en cada uno de los módulos (MTe y MTr). En cada parcela se enumeraron 10 plantas sobre las cuales se realizaron monitoreos de la mosca blanca T. vaporariorum y de la crisopa $C$. argentina, en las siguientes fechas: 24/09, 08/10, 28/10, 04/11, 15/11,
22/11, 29/11 y 10/12 (Figura 2). Se registró el número de adultos de mosca blanca por estrato (inferior, medio, superior) de planta. Cabe aclarar que se incluyó a $C$. argentina en los monitoreos preliberación porque al tratarse de una especie nativa cabía la posibilidad de que esté presente naturalmente en el cultivo, pudiendo esto interferir en el resultado de las liberaciones controladas de individuos de esta misma especie.

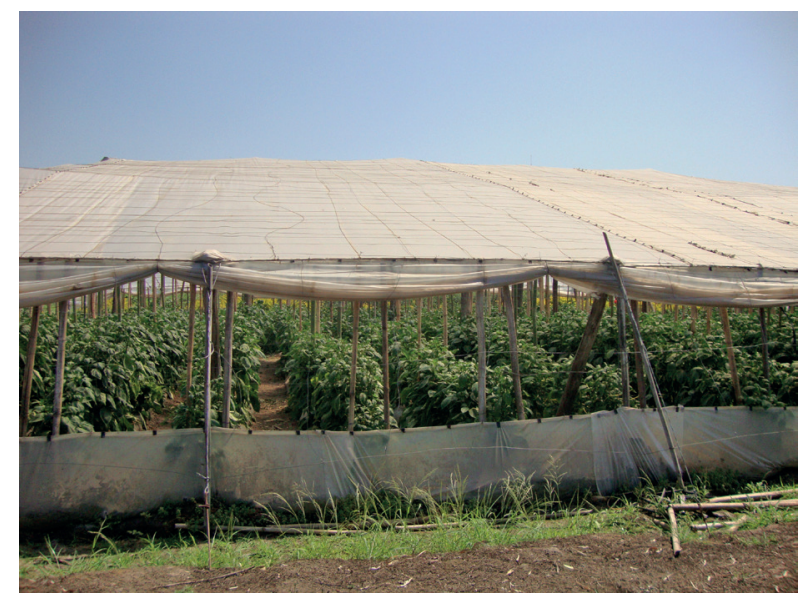

Figura 1. Invernáculo tipo parral de Almería con cultivo de pimiento (C. annuum) en Tucumán, Argentina.

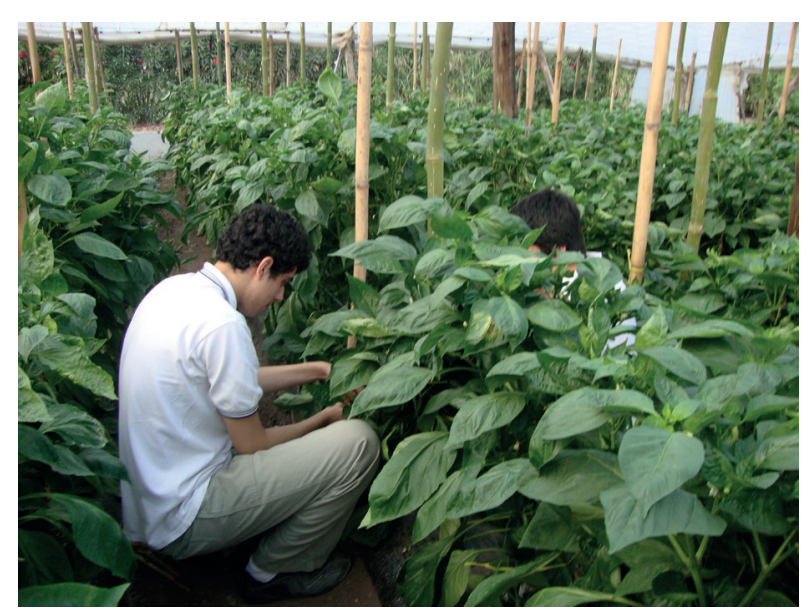

Figura 2. Monitoreo de T. vaporariorum en cultivo de pimiento bajo invernáculo.

Las liberaciones controladas de $C$. argentina se realizaron en las parcelas seleccionadas de MTr cuando el número de individuos de T. vaporariorum (Figura 3) por planta superó el umbral de daño económico, > 50 adultos por planta, basados en los resultados expuestos por Bellotti et al. (2007). 
Con los monitoreos preliberación, se determinó que eran necesarias dos liberaciones controladas de adultos y de larvas de $C$. argentina en las respectivas parcelas de MTr. Los adultos y larvas de $C$. argentina que se liberaron en el cultivo de pimiento provenían de una cría masiva realizada en cámaras del Centro de Investigaciones para la Regulación de Poblaciones de Organismos Nocivos (CIRPON), de Tucumán.

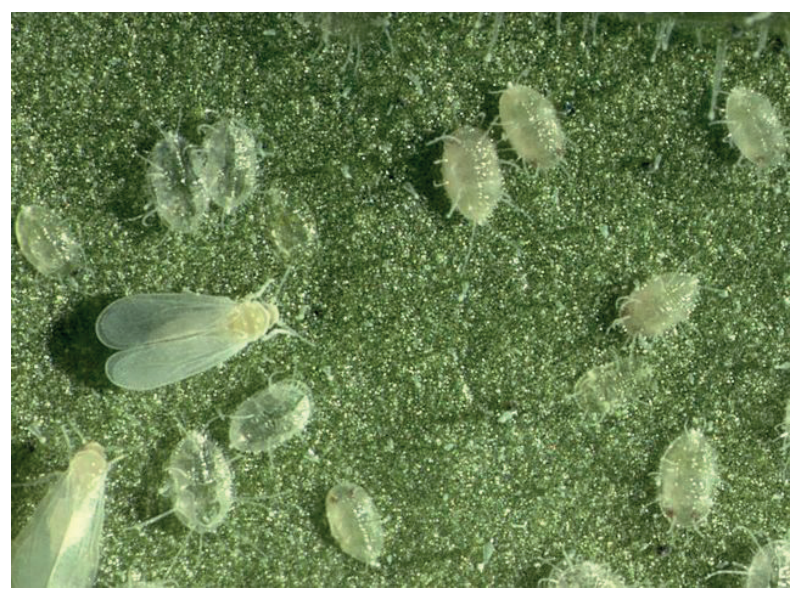

Figura 3. Adultos y ninfas de T. vaporariorum en hoja de pimiento.

La primera liberación de $C$. argentina se realizó el 23/11/2010, para este procedimiento se utilizó un esquema de 10 plantas alternadas por parcela de 20 plantas, esparciendo las larvas mezcladas con cascarilla de quínoa esterilizada sobre hojas (Figura 4). Se liberaron 150 adultos y 100 larvas (Figura 5) de crisopa en estadio L2, a razón de 1 a 2 larvas por planta, mientras que los adultos fueron liberados al azar, debido a su capacidad de dispersión. Adicionalmente, se colocaron recipientes de acrílico con una mezcla de atrayentes para adultos, mezcla de miel, polen y agua en relación $2: 2: 1$, con el fin de favorecer la permanencia de los insectos en el interior del invernáculo (Figura 6). El muestreo para evaluar el efecto de esta liberación se realizó el 29/11 en horas de la mañana.

La segunda liberación se realizó el 29/11 en horas de la tarde y constó de 100 individuos de $C$. argentina en estadio larval L3, a razón de 1 a 2 larvas por planta, siguiendo el mismo esquema de la primera liberación. El muestreo para evaluar el efecto de esta liberación se realizó el 10/12. En ambos muestreos, posteriores a la liberación, se eligieron 10 plantas al azar por cada parcela de 20 plantas. Se realizó la transformación logarítmica de los datos, análisis de la varianza y prueba t.

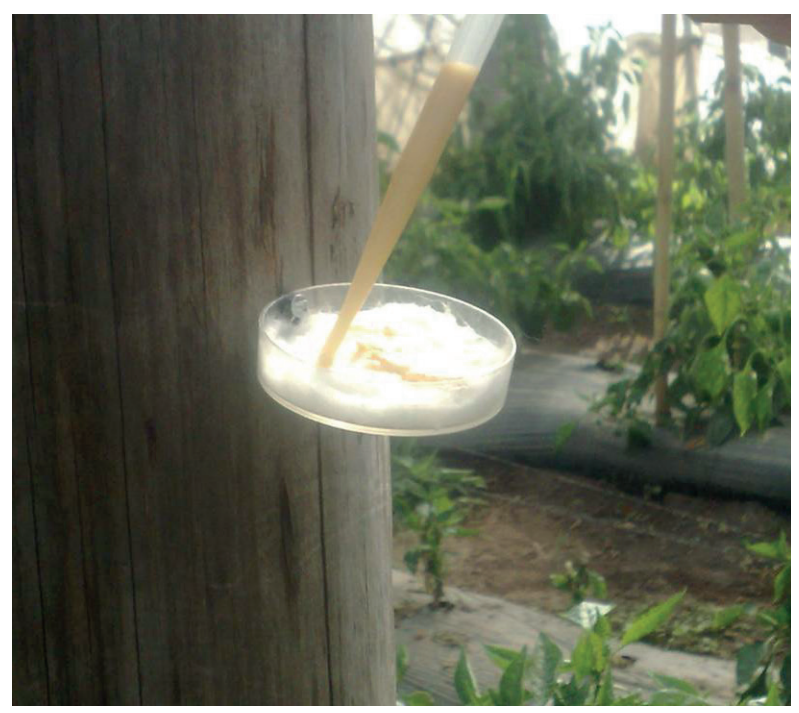

Figura 4. Método de liberación de $C$. argentina considerado en el estudio.

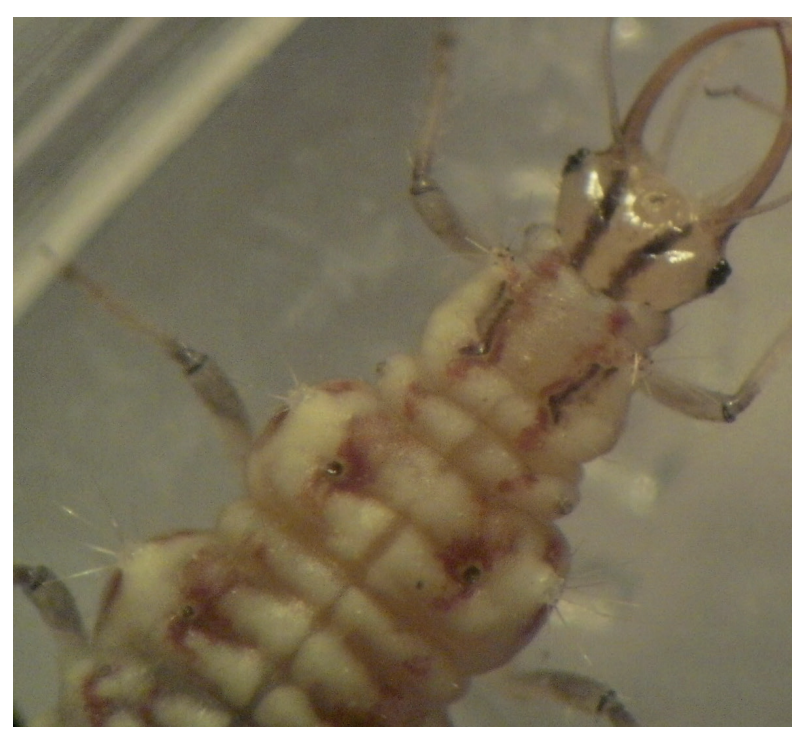

Figura 5. Chrysoperla argentina en su tercer estadio larval. El protorax con dos bandas laterodorsales delgadas de color pardo rojizo es un carácter morfológico de valor taxonómico (Foto cortesía de Carmen Reguilón). 


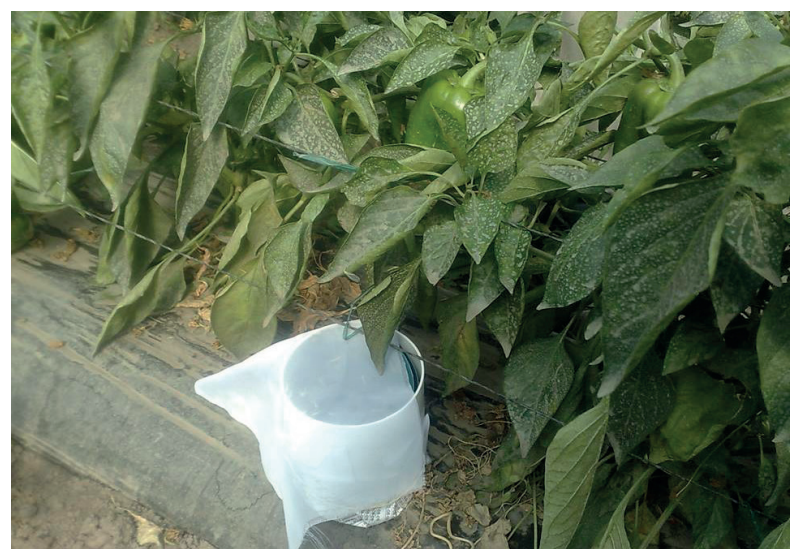

Figura 6. Estrategia utilizada como atrayente para C. argentina.

\section{RESULTADOS}

Los muestreos del 24/09 y el 08/10 no mostraron la presencia de individuos de T. vaporariorum en MTe 0 MTr (Tabla 1 y Figura 7). Desde el 28/10 se registraron los primeros ejemplares de la plaga en ambos sectores del invernadero (MTe y MTr), en cantidades similares en ambos ( 4,0 individuos/planta). No se observaron ni preferencias varietales, ni de ubicación en los diferentes estratos de la planta, por parte de T. vaporariorum. En los muestreos del 04/11 y 15/11, en ambos sectores por

Tabla 1. Promedio de adultos de T. vaporariorum por planta de pimiento (en invernadero) en los módulos testigo y tratamiento en diferentes fechas de muestreo (Tucumán, Argentina).

\begin{tabular}{|c|c|c|}
\hline $\begin{array}{l}\text { Fecha de } \\
\text { muestreo }\end{array}$ & $\begin{array}{l}\text { Promedio del } \mathrm{N}^{0} \text { de } \\
\text { adultos de Tv }{ }^{(1)} \text { por } \\
\text { planta en módulo } \\
\text { testigo }\end{array}$ & $\begin{array}{l}\text { Promedia del } \mathrm{N}^{0} \text { de } \\
\text { adultos de Tv por } \\
\text { planta en módulo } \\
\text { tratamiento }\end{array}$ \\
\hline 24-Sep & 0 & 0 \\
\hline 08-Oct & 0 & 0 \\
\hline 28-Oct & $3,85 \pm 5,09$ & $4,40 \pm 4,12$ \\
\hline 04-Nov & $14,05 \pm 10,11$ & $14,95 \pm 14,77$ \\
\hline $15-\mathrm{Nov}$ & $22,43 \pm 20,29$ & $21,50 \pm 12,15$ \\
\hline $22-\mathrm{Nov}^{(2)}$ & $70,13 \pm 7,68$ & $70,01 \pm 5,12$ \\
\hline 29-Nov ${ }^{(3)}$ & $73,20 \pm 6,79$ & $54,55 \pm 6,53$ \\
\hline 10-Dic & $146,05 \pm 17,36$ & $44,45 \pm 8,18$ \\
\hline
\end{tabular}

(1)Tv: Trialeurodes vaporariorum; (2) Primera liberación de crisopas en módulo tratamiento; (3) Segunda liberación de crisopas en módulo tratamiento. igual, el número de individuos de la plaga ascendió a $\sim 15$ y $\sim 22$ individuos/planta, respectivamente.

Las temperaturas máximas medias y mínimas medias dentro del invernáculo, desde que aparecieron las primeras moscas blancas $(28 / 10)$ hasta la última fecha de muestreo (10/12), fueron de $47,0 \pm 3,0{ }^{\circ} \mathrm{C}$ y $12,1 \pm 5,4$ ${ }^{\circ} \mathrm{C}$. En el caso de la humedad relativa ambiental, los valores fueron $87,0 \pm 6,9 \%$ y $11,0 \pm 1,7 \%$.

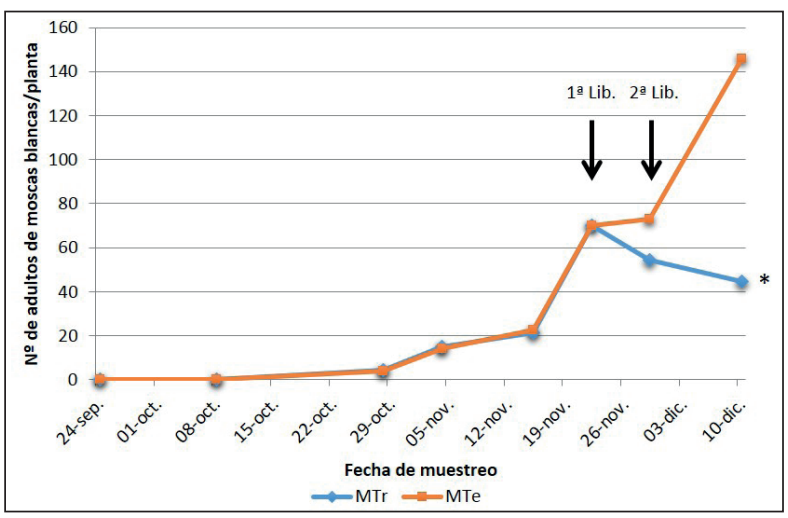

Figura 7. Evolución de las poblaciones de adultos de "la mosca blanca de los invernaderos" (T. vaporariorum) en dos sectores de un invernadero (MTe: módulo testigo sin tratar; MTr: módulo tratado con liberaciones controladas de la crisopa $C$. argentina) en Tucumán, Argentina. Las flechas indican los dos momentos en que se hicieron las liberaciones de $C$. argentina $\left(1^{\mathrm{a}} \mathrm{Lib}\right.$. 23/11; 2a Lib. 29/11). En la fecha 10/12, el “*” significa que entre las medias de MTe y MTr hay diferencias estadísticamente significativas a un nivel de $5 \%$ de probabilidad (p-valor según ANOVA). En todas las demás fechas, las diferencias entre las medias de MTe y MTr fueron estadísticamente no significativas al $5 \%$ de probabilidad.

Entre el muestreo del 22/11 con respecto al 15/11 se observó un alto incremento en el crecimiento de la población de T. vaporariorum. En este punto fue cuando la abundancia superó los 50 individuos/planta, es decir, que la densidad del fitófago superó el umbral de daño económico por igual en MTe y MTr (Figura 7). Cabe aclarar que hasta este momento no se había observado la presencia de $C$. argentina en ninguna de sus formas. Ante este aumento de los niveles poblacionales de la plaga, el 23/11 se realizó la primera liberación controlada del depredador, a los fines de reducir la densidad del fitófago en MTr. Cuando se hizo el primer 
muestreo posterior a la liberación, el 29/11, se observó un efecto de control de las crisopas sobre las moscas blancas, pero sin que la diferencia con el testigo sea estadísticamente significativa (MTr $=54,6$ y MTe $=72,2$ individuos/planta).

Debido a que la población de mosca blanca se mantenía por encima del umbral de daño económico ( $>50$ individuos/planta), se decidió hacer la segunda liberación controlada de crisopas ese mismo día. Posteriormente, el 10/12, para verificar el impacto de esta liberación sobre la plaga, se hizo un muestreo. Se registraron valores de individuos de mosca blanca en MTe de 146,05 y en MTr de 44,45 (Figura 7). El análisis de la varianza (Tabla 2), permitió determinar que esta diferencia era estadísticamente significativa ( $p$ > 0,01746), con lo cual se había alcanzado el objetivo de reducir la población de la plaga a niveles inferiores al umbral de daño económico. La presencia de huevos de $C$. argentina en el envés de las hojas de las plantas de pimiento permitió inferir que había posibilidades de permanencia del insecto benéfico en el cultivo por un tiempo más prolongado.

Con la Prueba "t" de Student, se confirmó que existen diferencias estadísticamente significativas entre las medias de las muestras testigo y tratamiento correspondientes al muestreo 10/12 (Tabla 3).

Tabla 2. Efecto de la liberación de $C$. argentina sobre el número de adultos de T. vaporariorum, en un cultivo de pimiento en invernadero en Tucumán, Argentina. Datos correspondientes a la fecha de muestreo 10/12/2010. Se presenta el valor P del análisis de varianza.

\begin{tabular}{lcc}
\hline & Testigo & Tratamiento \\
\hline $\begin{array}{l}\text { Media del nro. de adultos de } T . \\
\text { vaporariorum por planta }\end{array}$ & 146,05 & 44,45 \\
Probabilidad (Pr > F) & 0,01746 & \\
\hline
\end{tabular}

Tabla 3. Prueba "t” de Student para dos poblaciones, testigo y tratamiento (liberación de C. argentina), suponiendo varianzas desiguales. Los valores correspondientes al número de adultos de T. vaporariorum por planta fueron transformados logarítmicamente y con los valores transformados se calcularon las medias y las varianzas. Datos correspondientes a la fecha de muestreo 10/12/2010.

\begin{tabular}{lrr}
\hline & Testigo & Tratamiento \\
\hline Media & 2,161576381 & 1,640269011 \\
Varianza & 0,00268179 & 0,007290552 \\
$\mathrm{P}(\mathrm{T}<=\mathrm{t})$ dos colas & & $3,11336 \times 10^{-21}$ \\
\hline
\end{tabular}

\section{DISCUSIÓN}

Las liberaciones controladas de crisopas, especialmente las de larvas, permitieron establecer una relación depredador/presa de 1:50 (más eficiente que las relaciones encontradas para parasitoides) y una relación depredador (adulto)/planta de 1:3,6, con la cual se logró disminuir la población de la plaga en un 63,6 \% (de 70 a 44,5 individuos por planta), es decir, a niveles inferiores al umbral de daño económico. Otros enemigos naturales utilizados en diferentes investigaciones con fines de control biológico de ninfas de T. vaporariorum, en tomate bajo cubierta, fueron los parasitoides Encarsia tricolor Föerster (Arroyo et al., 1988) y E. formosa Gahan (Aragón et al., 2008), estas investigaciones registran reducciones de 50 y $80 \%$, respectivamente. Teniendo en cuenta que ambos parasitoides tienen preferencias por los estadios L3 y L4 de la plaga, las liberaciones se efectuaron ante bajas densidades poblacionales de mosca blanca, una vez que la primera generación de moscas alcanzó los mencionados estadios. Se determinó que la proporción de liberación, para alcanzar el mayor 
control, fue de 1:17 (parasitoide/presa) para E. tricolor y 1:3 para $E$. formosa, donde ambas relaciones mucho más elevadas que la alcanzada con $C$. argentina, lo cual significa que se necesitan más parasitoides por presa en comparación con las crisopas.

En evaluaciones de control de T. vaporariorum con extracto de ajo (Allium sativum), en cultivo de tomate bajo cubierta, se verificaron reducciones de la población de adultos pero no de ninfas, decreciendo su efecto en los sucesivos recuentos (Strassera et al., 2007). En nuestro estudio, el control sobre la mosca blanca fue más amplio y por su magnitud se extendió a todos los estados de la plaga. Se podría prolongar la permanencia de las crisopas en el cultivo y distribuir la mezcla atrayente en el interior del invernáculo.

\section{CONCLUSIONES Y RECOMENDACIONES}

La reducción de la población de T. vaporariorum, con liberaciones controladas del depredador $C$. argentina en cultivo de pimiento bajo invernadero se logró satisfactoriamente. Al tratarse de un entomófago generalista, $C$. argentina no tiene preferencias por los estados de desarrollo de la mosca blanca, por lo que ejerce una intensa y constatable depredación sobre todos ellos. El umbral de liberación se estableció de manera independiente al fenómeno de interferencia, por lo que no necesariamente requieren bajas densidades poblacionales de la plaga para la efectividad del control.

Otras importantes ventajas de $C$. argentina son, por un lado la factibilidad de su cría masiva a través de técnicas sencillas, y por el otro, al tratarse de una especie nativa, consigue una rápida adaptación y permanencia en la vegetación circundante una vez levantado el cultivo. Para concluir los resultados de este estudio, se puede afirmar que $C$. argentina es un enemigo natural promisorio contra T. vaporariorum, en cultivos de pimiento en invernadero en regiones subtropicales del norte del país. Sería interesante conocer el tiempo de permanencia de $C$. argentina en el cultivo más allá del período estudiado, teniendo en cuenta que en diciembre comienza el verano en Argentina y las temperaturas dentro del invernáculo pueden alcanzar valores restrictivos para el normal cumplimiento del ciclo de vida de las crisopas. De ese tipo de estudios, o de otros más específicos, se podría obtener información acerca de la adaptabilidad de $C$. argentina a los climas tropicales, de las extensas zonas hortícolas de las provincias de
Salta y Jujuy, colindantes con Bolivia. También sería importante verificar la acción depredadora de esta especie de crisópido, sobre otras plagas y en otras solanáceas que se cultivan en invernáculo en la región, tales como tomate y berenjena (Solanum melongena $\mathrm{L}$.).

\section{AGRADECIMIENTOS}

A la Téc. Fitosanitaria Noelia Ramos y los alumnos Herman Emge y Álvaro Chico Costas, por la colaboración en el trabajo de campo, y a la Ing. Agr. Claudia Funes, por la revisión crítica del manuscrito. Este trabajo fue financiado por el Proyecto Regional Tucumán Norte (TUSGO 311001 INTA- Famaillá) y por el Proyecto "Neurópteros del norte argentino: Biología y capacidad depredadora aplicada a cultivos de importancia agroeconómica” (Fundación Miguel Lillo).

\section{BIBLIOGRAFÍA}

Aragón, S., Rodríguez, S. y Cantor, F. 2008. Criterios de liberación de Encarsia formosa Gahan (Hymenoptera: Aphelinidae) para el control de Trialeurodes vaporariorum (Westwood) (Hemiptera: Aleyrodidae) en tomate. Agronomía Colombiana 26(2): 277-284.

Arroyo, M., Castresana, L., Notario-Gómez, A. 1988. Control biológico de la mosca blanca de los invernaderos, Trialeurodes vaporariorum WEST (Hemiptera: Aleyrodidae) por Encarsia tricolor FOERS (Hymenoptera: Aphelinidae) en tomate de invernadero. Boletín Sanidad Vegetal - Plagas 14(3): 447-459.

Ávila, A.N., Arias, M.J. y Ortega, M.E. 2009. Ingesta de huevo de mosca blanca por Chrysoperla argentina. Resúmenes de la III Jornada de Jóvenes Investigadores 2009. Tucumán, Argentina.

Aybar-Guchea, M., Herrero, M.I., Aralde, M.R. y Taboada D. 2010. Dos sistemas de manejo sobre insectos benéficos de Bemisia tabaci (Gennadius) en un cultivo de pimiento (Capsicum annuum L.) bajo cubierta. Resúmenes de la IV Jornada de Jóvenes Investigadores 2010. Tucumán, Argentina.

Bale, J.S., van Lenteren, J.C. y Bigler, F. 2008. Biological control and sustainable food production. Philosophical Transactions of the Royal Society B 363: 761-776.

Bellotti, A.C., Arias, B., Herrera, C.J. y Holguín, C.M. 2007. Manejo integrado de moscas blancas asociadas al cultivo de la yuca. Publicación CIAT No 358. Colombia. 
Bisset, J.A. 2002. Uso correcto de insecticidas: control de la resistencia. Revista Cubana de Medicina Tropical 54(3): 202-219.

Breene, R.G., Meagher, R.L., Nordlund, D.A. y Wang, Y.T. 1992. Biological control of Bemisia tabaci (Homoptera: Aleyrodidae) in a greenhouse using Chrysoperla rufilabris (Neuroptera: Chrysopidae). Biological Control 2(1): 9-14.

Brimmer, T. y Boland, G. 2003. A review of the non-target effects of fungi used to biologically control plant diseases. Agriculture Ecosystems and Environment 100: 1-16.

Dennehy, T.J., Degain, B.A., Harpold, V.S., Brown, J.K., Morin, S., Fabrick, J.A., Byrne, F.J. y Nichols, R.L. 2005. New challenges to management of whitefly resistance to insecticides in Arizona. In: University of Arizona Cooperative Extension, Vegetable Report. (http://cals. arizona.edu/pubs/crops/az1382/az1382_2.pdf)

Duffus, J.E. 1965. Beet pseudo-yellows virus, transmitted by the greenhouse whitefly (Trialeurodes vaporariorum). Phytopathology 55(4): 450-453.

Fernández, J.A., Ghiggia, L.I., Macián, A.J., Jaime, A.P. y Arce, O.E. 2009. Chrysoperla argentina González Olazo y Reguilón (Neuroptera-Chrysopidae) agente de control de Bemisia tabaci (Gennadius) en cultivar de pimiento (Capsicum annuum L.) bajo carpa en Tucumán. Resúmenes de las XIII Jornadas Fitosanitarias Argentinas 2009. Las Termas de Río Hondo, Santiago del Estero, Argentina.

Fernández-Lozano, J. 2012. La producción de hortalizas en Argentina. Secretaría de Comercio Interior. Corporación del Mercado Central de Buenos Aires. http://www. mercadocentral.gob.ar Consulta: Marzo de 2014

Gerling, D., Kravchenko, V. y Lazare, M. 1997. Dynamics of common green lacewing (Neuroptera: Chrysopidae) in Israeli cotton fields in relation to whitefly (Homoptera: Aleyrodidae) populations. Environmental Entomology 26(4): 815-827.

Ghiggia, L.I., Fernández, R.V., Yasem de Romero, M.G. y Fernández, J.A. 2003. Cladosporium sp. sobre Dialeurodes citrifolii (Morgan) (Homoptera-Aleyrodidae) en plantaciones de limonero de la provincia de Tucumán. Revista Agronómica del Noroeste Argentino 31 (1-4): 27-30.

Haramboure, M., Reguilón, C., Alzogaray, R.A. y Schneider, M.I. 2014. First record of Chrysoperla asoralis and C. argentina in horticultural fields of La Plata associated with the sweet pepper (Capsicum annuum L.). Revista de la Sociedad Entomológica Argentina 73(3-4): 187-190.

López, S.N., Viscarret, M.M., Andorno, A.V. y Botto, E. 2005. Estudio de la interacción entre Encarsia formosa y Eretmocerus corni (Hymenoptera: Aphelinidae), parasitoides de la mosca blanca de los invernáculos Trialeurodes vaporariorum (Hemiptera: Aleyrodidae). Revista de Investigaciones Agropecuarias INTA 34(3): 73-82.

Macián, A.J., Ghiggia, L.I., Fernández, J.A. y Jaime, A.P. 2009. Eficacia de control de Neem (Azaderachta indica L.) y Chrysoperla argentina González Olazo y Reguilón (Neuroptera-Chrysopidae) en cultivar de pimiento (Capsicum annuum L.) bajo carpa en Lules, Tucumán. Horticultura Argentina 28(67): 150.

MAG Costa Rica. 2015. Guía técnica para el monitoreo de moscas blancas. Servicio Fitosanitario del Estado.

Ortiz-Catón, M., Medina-Torres, R., Valdivia-Bernal, R., Ortiz-Catón, A., Alvarado-Casillas, S. y Rodríguez-Blanco, J.R. 2010. Mosquitas blancas, plaga primaria de hortalizas en Nayarit. Revista Fuente 2(5): 31-40.

Polack, L.A. 2005. Manejo integrado de moscas blancas. Boletín Hortícola Nº 31. EEA San Pedro INTA. Argentina.

Reguilón, C., Correa, M. del V., Yapur, A. y Kirschbaum, D.S. 2014. Evaluación del efecto de la liberación de Chrysoperla externa y C. argentina (Neuroptera: Chrysopidae) como agentes de control biológico de trips en cultivo de frutilla en Tucumán. Horticultura Argentina 33(82): 56.

Reguilón, C., González-Olazo, E.V. y Núñez-Campero, R.S. 2006. Morfología de los estados inmaduros de Chrysoperla argentina (Neuroptera: Chrysopidae). Acta Zoológica Lilloana 80(1-2): 31-39.

Ruiz, J. y Medina, Z.J. 2001. Avances en el manejo integrado de Bemisia tabaci en tomate y chile en Oaxaca, México. Manejo Integrado de Plagas 59: 34-40.

Sistema Nacional Argentino de Vigilancia y Monitoreo de Plagas. 2015. Trialeurodes vaporariorum. http://www. sinavimo.gov.ar/plaga/trialeurodes-vaporariorum

Strassera, M.E., Polack, L.A., Mezquiriz, N. y Martinez Quintana, O.R. 2007. Evaluación de diferentes tratamientos para el control de la "mosca blanca de los invernáculos" Trialeurodes vaporariorum (Westwood) (Homoptera: Aleyrodidae) en tomate bajo cubierta en el Cinturón Hortícola Platense. Horticultura Argentina 26(61): 116. 
Tapia, S., Leaño M., Agostini, E. y Álvarez, S. 2005. Relevamiento de moscas blancas (Hemiptera, Aleyrodidae) y sus biocontroladores en cultivos de tomate (Licopersicon esculentum Mill.) pimiento (Capsicum annuum L.) y berenjena (Solanum melongena L.), de Jujuy y Salta. Resúmenes del VI Congreso Argentino de Entomología 2005. Tucumán, Argentina.

Tauber, M.J., Tauber, C.A., Daane, K.M. y Hagen, K.S. 2000. Commercialization of predators: recent lessons from green lacewings (Neuroptera: Chrysopidae: Chrysoperla). American Entomologist 46: 26-38.

Velázquez-Grisales, L.P. 2004. Estudio de la biología de Ceraeochrysa claveri (Neuroptera: Chrysopidae) alimentada con dos tipos de presa en condiciones de laboratorio. Informe científico. Centro Internacional de Agricultura Tropical y Universidad de Caldas, Facultad de Ciencias Agropecuarias. Manizales, Colombia.

Fecha de recepción: 27/06/2015

Fecha de aceptación: 13/11/2015

Para citar este artículo: Flóres, G.C., C. Reguilón, G.L. Alderete, D.S. Kirschbaum. 2015. Liberación de Chrysoperla argentina (Neuroptera: Chrysopidae) para el control de Trialeurodes vaporariorum (Westwood) (Hemiptera, Aleyrodidae) en invernáculo de pimiento en Tucumán, Argentina. Revista Intropica Vol. 10: 28 - 36 